DRAFT VERSION OCTOBER 25, 2018

Preprint typeset using $\mathrm{LT}_{\mathrm{E}} \mathrm{X}$ style emulateapj v. 4/12/04

\title{
THE VOID PHENOMENON EXPLAINED
}

\author{
JEREMY L. TINKER ${ }^{1} \&$ CHARLIE CONROY ${ }^{2}$ \\ ${ }^{1}$ Kavli Institute for Cosmological Physics \& Department of Astronomy and Astrophysics, University of Chicago \\ ${ }^{2}$ Department of Astrophysical Sciences, Princeton University, Princeton, NJ 08544, USA \\ Draft version October 25, 2018
}

\begin{abstract}
We use high-resolution N-body simulations, combined with a halo occupation model of galaxy bias, to investigate voids in the galaxy distribution. Our goal is to address the "void phenomenon" of Peebles (2001), which presents the observed dearth of faint galaxies in voids as a challenge to the current cosmology. In our model, galaxy luminosity is determined only as a function of dark matter halo mass. With this simple assumption, we demonstrate that large, empty voids of $\sim 15 \mathrm{~h}^{-1} \mathrm{Mpc}$ in diameter are expected even for galaxies seven magnitudes fainter than $L_{*}$. The predictions of our model are in excellent agreement with several statistical measures; $(i)$ the luminosity function of galaxies in underdense regions, $(i i)$ nearest neighbor statistics of dwarf galaxies, (iii) the void probability function of faint galaxies. In the transition between filaments and voids in the dark matter, the halo mass function changes abruptly, causing the maximum galaxy luminosity to decrease by $\sim 5$ magnitudes over a range of $\sim 1 h^{-1} \mathrm{Mpc}$. Thus the boundary between filaments and voids in the galaxy distribution is nearly as sharp for dwarfs as for $\sim L_{*}$ objects. These results support a picture in which galaxy formation is driven predominantly by the mass of the host dark matter halo, and is nearly independent of the larger-scale halo environment. Further, they demonstrate that $\Lambda \mathrm{CDM}$, combined with a straightforward bias model, naturally predicts the existence of the void phenomenon.
\end{abstract}

Subject headings: galaxies: halos — large scale structure of the universe

\section{INTRODUCTION}

Galaxy redshift surveys have revealed a complex network of clusters, filaments and walls. They have also demonstrated that the expansive regions between these structures are nearly bereft of galaxies (see Gregory \& Thompson 1978; Kirshner et al. 1981; Vogeley et al. 1994 for early work, and Hoyle \& Vogeley|2004; Croton et al.|2004; Conroy et al. 2005; Patiri et al. 2006a; Tinker et al. 2007a for results from recent large-scale surveys). The surprisingly large size of voids - up to $\sim 30 h^{-1} \mathrm{Mpc}$ in diameter-and their apparent depth, in terms of their luminosity density, have begged questions about their formation mechanism. Why do faint galaxies avoid the voids defined by their brighter brethren? Further, the few objects found in and near underdense regions appear to represent a fair sample of the overall galaxy population. Peebles (2001, hereafter P01) presented these observations as "the void phenomenon". The straightforward argument is that the deepest voids in the dark matter distribution predicted by standard $\Lambda \mathrm{CDM}$ are never completely empty; they still contain mass and significant numbers of low-mass halos. Therefore, voids should be the preferable environment of faint dwarf galaxies. However, P01 demonstrates that dwarf galaxies tend to be distributed similarly to brighter spirals, and the local void exhibits a paucity of dwarfs. Peebles (2007) further shows that the edge of the local void has the same sharp edge for both bright and faint galaxies. If halos exist in voids, then where are the galaxies?

In this paper we present a quantitative model of galaxy bias that allows us to directly address this question. We use the Halo Occupation Distribution (HOD; e.g. Seliak 2000; Scoccimarro et al. 2001; Cooray \& Sheth 2002; Berlind \& Weinberg 2002) to specify the relationship between galaxies and dark matter. In the HOD framework, this relationship is quantified by $P(N \mid M)$, the probability that a halo of mass $M$ contains $N$ galaxies of a given sample. Galaxy samples can be defined by luminosity, color, star formation rate, or any other galaxy property. For each sample, $P(N \mid M)$ will be different but can be calibrated with observational data. The HOD has emerged as the dominant tool for interpreting clustering measurements for a wide range of redshifts and galaxy classes (see, for a tip of the iceberg, Zehavi et al. 2004, 2005; Zheng et al. 2007; van den Bosch et al. 2007; Tinker et al.|2007b a; Chen 2007; White et al. 2007; Padmanabhan et al. 2008). The results obtained from analysis of observational data are in excellent agreement with the results of semi-analytic models, hydrodynamic cosmological simulations, and high-resolution collisionless simulations (Kravtsov et al. 2004; Zheng et al. 2005; Conroy et al. 2006). Our knowledge of the mapping between galaxies and halos is now well-established and can be confidently extended into regimes in which observational data are lacking. Here we take HOD results calibrated on observed galaxy samples that occupy $\gtrsim 10^{11} h^{-1} \mathrm{M}_{\odot}$ halos and extrapolate to lower masses in order to model the distribution of dwarf galaxies. We will demonstrate that the predictions of this model are in excellent agreement with the observations of P01: deep, wide voids are expected within hierarchical structure formation, even for the faintest of galaxies.

One of the difficulties in directly addressing the void phenomenon is that the problem has not been clearly defined. The definition of a dwarf galaxy varies in the literature, and the predictions of $\Lambda \mathrm{CDM}$ in this regime have not been robustly specified. To elucidate the problem, P01 compared the spatial distribution of "ordinary" galaxies to samples of "test" dwarf galaxies within the Optical Redshift Survey (ORS; Santiago et al. 1995). The number density of the ordinary sample is comparable to the number density of $M_{r}<-16$ galaxies $^{1}$ in the Sloan Digital Sky Survey (SDSS; Blanton et al. 2005). Thus to give our model a fixed target we

1 For brevity, all galaxy magnitudes assume a Hubble constant $h \equiv$ $H_{0} / 100=1$. All $r$-band magnitudes have been $k$-corrected to $z=0$, as with the Blanton et al. (2005) luminosity function. 
stipulate that the void phenomenon begins at galaxies fainter than $M_{r}=-16$, roughly Small Magellanic Cloud-type galaxies and fainter.

In Tinker et al. (2007a) we used the HOD to make predictions for void probability statistics for galaxies brighter than $M_{r}=-19$. Our predictions were in excellent agreement with our measurements from SDSS Data Release 4 (Adelman-McCarthy et al. 2006). Thus we concluded that there is no conflict between the observed voids and those predicted by $\Lambda \mathrm{CDM}$ for galaxies down to this magnitude (roughly $0.2 L_{*}$, given an $M_{*}$ of -20.44 from Blanton et al. 2003). More importantly for the model presented herein, the results of Tinker et al. (2007a) support a model in which the luminosities of galaxies are determined entirely by the mass of the host halo, independent of the environment in which the halo formed. This allows us to robustly predict the distribution of void galaxies within a model that connects galaxies with halos through the halo mass only.

Several other studies have investigated galaxy voids and void galaxies through a variety of techniques. Mathis \& White (2002) and Benson et al. (2003), using semi-analytic models, concluded that galaxies of $M_{r} \sim-18.5$ avoid the voids of the brighter galaxies. These studies were limited by numerical resolution and were not able to model the dwarf galaxies on which P01 based his argument. Furlanetto \& Piran (2006) constructed an analytic framework within which to explore the dependence of voids on galaxy luminosity, building on earlier work modeling voids in the dark matter by Sheth \& van de Weygaert (2004). However, no tests with numerical simulations or comparison to observational results were made. The model of Furlanetto \& Piran (2006) is an excellent tool for understanding the trends seen in the data, but detailed analysis of voids requires a simulation to produce the correct halo distribution. As we will show, the change in the halo mass function along the edge of a halo is of critical importance in the resulting void distribution. Additionally, observational data are all measured in redshift space, which is not incorporated into these analytic models.

This paper is organized as follows: In $\$ 2$ we present our HOD model and the numerical simulations employed to create mock galaxy distributions. In $\S 3$ we present the predicted void distribution, making quantitative comparisons to several sets of observational data: (a) the luminosity function of void galaxies, which has been measured down to $M_{r}=-14$ by Hoyle et al. (2005), (b) the nearest neighbor statistics of P01, which probe galaxies fainter than $M_{r}=-16$, and (c) the void probability function of galaxies down to $M_{r}=-17$, for which we present new measurements from SDSS Data Release 6 (Adelman-McCarthy et al. 2007). In $\$ 4$ we discuss our results. In all calculations, we assume a flat, $\Lambda \mathrm{CDM}$ universe with $\left(\Omega_{m}, \sigma_{8}, h, n, \Omega_{b}\right)=(0.3,0.9,0.7,1.0,0.04)$.

\section{METHODS}

\subsection{Halo Occupation Models}

Halo occupation models generally contain two important mass scales. The first is the mass at which halos become sufficiently large to host a single galaxy brighter than a specified luminosity threshold. The second is the mass at which halos become massive enough to host additional satellite galaxies. Results from both theory and observation have demonstrated that these masses scale in a straightforward fashion with galaxy luminosity, allowing for confident extrapolation to the dwarf galaxies on which we focus.
TABLE 1

Minimum Halo Mass as a FunCtion of $r$-BAND MAGNITUDE THRESHOLD

\begin{tabular}{cccccc}
\hline \hline$M_{r}$ & $\bar{n}_{g}$ & $M_{\min }$ & $M_{r}$ & $\bar{n}_{g}$ & $M_{\min }$ \\
\hline-22 & $2.63 \times 10^{-5}$ & 14.16 & -15 & $1.30 \times 10^{-1}$ & 10.60 \\
-21 & $1.23 \times 10^{-3}$ & 12.73 & -14 & $2.12 \times 10^{-1}$ & 10.38 \\
-20 & $6.59 \times 10^{-3}$ & 11.97 & -13 & $3.43 \times 10^{-1}$ & 10.16 \\
-19 & $1.58 \times 10^{-2}$ & 11.57 & -12 & $5.57 \times 10^{-1}$ & 9.94 \\
-18 & $2.88 \times 10^{-2}$ & 11.29 & -11 & $9.01 \times 10^{-1}$ & 9.72 \\
-17 & $4.83 \times 10^{-2}$ & 11.05 & -10 & $1.46 \times 10^{-0}$ & 9.51 \\
-16 & $7.95 \times 10^{-2}$ & 10.82 & & & \\
& & & & &
\end{tabular}

Note. - Galaxy densities are in units of
$\left(h^{-1} \mathrm{Mpc}\right)^{-3}$. All masses are in units of $h^{-1} \mathrm{M}_{\odot}$.

Functionally, the HOD breaks down the occupation of galaxies within halos into two distinct components: central galaxies and satellite galaxies. We use the standard parameterization for central galaxies brighter than a given magnitude limit:

$$
\left\langle N_{\text {cen }}\right\rangle_{M}=\frac{1}{2}\left[1+\operatorname{erf}\left(\frac{\log M-\log M_{\min }}{\sigma_{\log M}}\right)\right],
$$

where $M_{\min }$ is the mass at which a halo has a $50 \%$ probability of having a central galaxy above the defined luminosity threshold, and $\sigma_{\log M}$ is the width of the transition between 0 and 1 galaxies, physically representing the scatter of mass at fixed luminosity. The number of satellite galaxies scales as a power of the host mass, with a cutoff scale set by the central occupation function,

$$
\left\langle N_{\mathrm{sat}}\right\rangle_{M}=\left\langle N_{\mathrm{cen}}\right\rangle_{M} \times\left(\frac{M}{M_{1}}\right)^{\alpha_{\mathrm{sat}}}
$$

where $M_{1}$ is the mass scale at which a halo has, on average, one satellite brighter than the defined magnitude limit. The inclusion of $\left\langle N_{\text {cen }}\right\rangle_{M}$ in equation (2) ensures that the central galaxy is the brightest galaxy within a halo. The forms of these equations are in good agreement with the results of semi-analytic models of galaxy formation (Zheng et al.2005), high-resolution collisionless N-body simulations that resolve substructure (Kravtsov et al. 2004; Conrov et al. 2006), hydrodynamic simulations (Zheng et al. 2005), and analytic models of halo substructure (Zentner et al. 2005).

It has been demonstrated that the parameters of the occupation functions are nearly self-similar with luminosity, i.e., that $\alpha_{\text {sat }} \approx 1$ and $M_{1} / M_{\min } \approx 20$. These results have been found both in the theoretical results listed above and analyses of clustering measurements from numerous surveys at multiple redshifts (Zehavi et al. 2005; Zheng et al. 2007; Tinker et al. 2007b; van den Bosch et al. 2007). High-resolution N-body simulations have demonstrated that the subhalo mass function is nearly self-similar with parent halo mass, in good agreement with the HOD results obtained from observations (Kravtsov et al. 2004; Gao et al. 2004; De Lucia et al. 2004). These results extend down to subhalos of $M \sim 10^{7} h^{-1} \mathrm{M}_{\odot}$ (Diemand et al. 2007). With the now well-established connection between satellite galaxies and substructure within dark matter halos (Kravtsov et al. 2004; Conroy et al. 2006; Weinberg et al. 2006), the self-similarity of the HOD with luminosity is expected to extend far down the mass function to halos that host dwarf galaxies. In addition, because we are 
TABLE 2

Properties OF THE Simulation SET

\begin{tabular}{ccccc}
\hline \hline$L_{\text {box }}$ & $\epsilon\left[h^{-1} \mathrm{kpc}\right]$ & $N_{p}$ & $m_{p}\left[h^{-1} \mathrm{M}_{\odot}\right]$ & $M_{r}$ \\
\hline 384 & 14 & $1024^{3}$ & $4.39 \times 10^{9}$ & -20 \\
192 & 4.9 & $1024^{3}$ & $5.89 \times 10^{8}$ & -17 \\
96 & 1.4 & $1024^{3}$ & $6.86 \times 10^{7}$ & -14
\end{tabular}

Note. $-L_{\text {box }}$ is in units of $h^{-1}$ Mpc. $\epsilon$ is the Plummer force softening length. $N_{p}$ is the total number of particles in the simulation. Magnitudes in column 5 represent the lowest magnitude bin modeled by each simulation.

interested in voids, the details of the $\left\langle N_{\text {sat }}\right\rangle_{M}$ are largely irrelevant; as long as the fraction of galaxies that are satellites is roughly correct, the distribution of voids is not affected by the details of $\left\langle N_{\text {sat }}\right\rangle_{M}$ (Conroy et al. 2005; Tinker et al. 2006).

For each luminosity threshold, we set the value of $M_{\min }$ by matching the space density of galaxies from the Blanton et al. (2005) luminosity function, ie,

$$
\int_{M_{r}}^{\infty} \Phi\left(M_{r}\right) d M_{r}=\int_{0}^{\infty}\left(\left\langle N_{\text {cen }}\right\rangle_{M}+\left\langle N_{\text {sat }}\right\rangle_{M}\right) \frac{d n}{d M} d M
$$

where $d n / d M$ is the halo mass function, for which we use Tinker et al. (2008). The Blanton et al. (2005) result is accurate down to $M_{r}<-12$ galaxies. We set $M_{1}=22 M_{\min }$ and $\alpha_{\text {sat }}=1$ for all samples. For $\sigma_{\log M}$ we choose a value of 0.15 , which is consistent with the results of Tinker et al. (2007a) for $M_{r}<-19$ samples. This is somewhat smaller than that obtained by van den Bosch et al. (2007), but we note that the scatter between mass and luminosity is expected to be larger for blue-selected galaxy samples (which van den Bosch et al. 2007 analyze). Tinker et al. (2006) concluded that the values of $\sigma_{\log M}$ ranging from 0 to 0.5 produced little effect on the void distribution. We will test the dependence of our results on $\sigma_{\log M}$ in $\S 3$. Table 1 lists the value of $M_{\min }$ for each luminosity threshold.

\subsection{Numerical Simulations}

To investigate the distribution of voids and void galaxies with our HOD models, we populate a series of high-resolution N-body simulations of various box sizes kindly provided my M. Warren. The parameters of the simulations are listed in Table 2, and we will refer to each simulation by its box size in $h^{-1}$ Mpc: L96, L192, and L384. The simulations allow us to probe halos robustly down to $\sim 10^{10} h^{-1} \mathrm{M}_{\odot}$, the minimum mass scale of -12 galaxies. These simulations were performed using the hashed oct-tree code of Warren \& Salmon (1993), and were described in Warren et al. (2006). We use the spherical overdensity halo finder of Tinker et al. (2008) to identify halos in the simulation, defining halos with an overdensity $\Delta=200$ with respect to the background matter density. The mass resolution of the highest resolution simulation is 18 times higher than the Millennium Simulation of Springel et al. (2005), and 75 times higher than the simulation coupled to the semi-analytic galaxy formation model of Mathis \& White (2002).

To populate each simulation with galaxies, we use a Monte Carlo approach based on equations (1) and (2), assuming a nearest integer distribution for central galaxies and a Poisson distribution of satellite galaxies. The nearest integer approach is standard for central galaxies due to the limit of one central galaxy per halo. Poisson statistics provide an excellent description of the distribution of satellites in simulations (Kravtsov et al. 2004; Zheng et al. 2005) and in observations (Lin et al. 2004). By considering the HOD parameters as a function of luminosity, the full conditional luminosity function of each halo can be easily determined. The number of centrals in a bin $M_{r}$ to $M_{r}+\Delta M_{r}$ is $\left\langle N_{\text {cen }}\right\rangle_{M}^{\left(M_{r}\right)}-\left\langle N_{\text {cen }}\right\rangle_{M}^{\left(M_{r}+\Delta M_{r}\right)}$. The number of satellite galaxies in a magnitude bin is calculated in the same manner.

Central galaxies are placed at the center of the halo, and satellite galaxies are distributed randomly with the density profile of Navarro et al. (1997), with the concentration-mass relation of Bullock et al. (2001) (using the updated parameters of the model listed in Wechsler et al. 2006). Satellite velocities, relative to the halo motion, are taken from a Gaussian distribution with 1-dimensional dispersion of $\sigma^{2}=G M_{200} / 2 R_{200}$. We populate each simulation down to a limit of 100 particles per halo. The $384 h^{-1} \mathrm{Mpc}$ simulation is ideal for $M_{r}<-20$ samples, the $192 h^{-1} \mathrm{Mpc}$ sample resolves $M_{r}<-17$ samples, while the $96 h^{-1} \mathrm{Mpc}$ simulation probes galaxies as faint as $M_{r}=-14$. Although $M_{\min }$ for $M_{r}=-12$ is 127 particles, we must resolve halos below $M_{\min }$ to fully account for the scatter between mass and luminosity.

\section{RESULTS}

\subsection{The Cosmic Web in the Faintest Galaxies}

Figure 1 presents a slice through L96 that is typical of the structure in these simulations. The galaxy positions are in redshift space, assuming the distant-observer approximation with the $y$-axis being the line of sight. Along the filaments, $L_{*}$ and dwarf galaxies cohabitate, along with all luminosities in between. The transition between filaments and voids is very sharp, however. Deep voids of $\sim 10 \mathrm{~h}^{-1} \mathrm{Mpc}$ in diameter, empty of even the faintest resolved galaxies, are not uncommon in this simulation. The deepest void, centered at $(x, y)=(80,48) h^{-1} \mathrm{Mpc}$, is nearly $18 h^{-1} \mathrm{Mpc}$ along its longest axis. The transition from the main filament in the slice (running along the $y$-axis) into the void on either side is sharp for both bright and faint galaxies, resembling the galaxy distribution around the local void shown in Peebles (2007). Around the local void, between 5 to $7 h^{-1} \mathrm{Mpc}$ from the void center, the number of galaxies $M_{B}<-18$ and $M_{B}>-18$ drops essentially to zero. The abrupt transition from filament to void is ubiquitous in the cosmic web, even for galaxies hosted by $10^{10} h^{-1} \mathrm{M}_{\odot}$ halos. We note that halo velocities are usually directed along the axis of the filament, thus choosing the $x$ axis as the line of sight does not noticeable change the galaxy distribution plotted in Figure 1

\subsection{The Luminosity Function of Void Galaxies}

The most straightforward quantifiable test of our model is the number of galaxies located within voids, and their distribution as a function of luminosity. Hoyle et al. (2005) created a sample of "void" galaxies in the SDSS by identifying galaxies with local densities below a critical value of $\delta \equiv \delta \rho / \bar{\rho}=-0.6$ in a top-hat sphere of $7 h^{-1}$ Mpc. Densities were calculated with respect to galaxies with $M_{r}<-20.5$. We have constructed a sample of void galaxies in our simulations following these same criteria. All densities are calculated in redshift space assuming the distant observer approximation. 


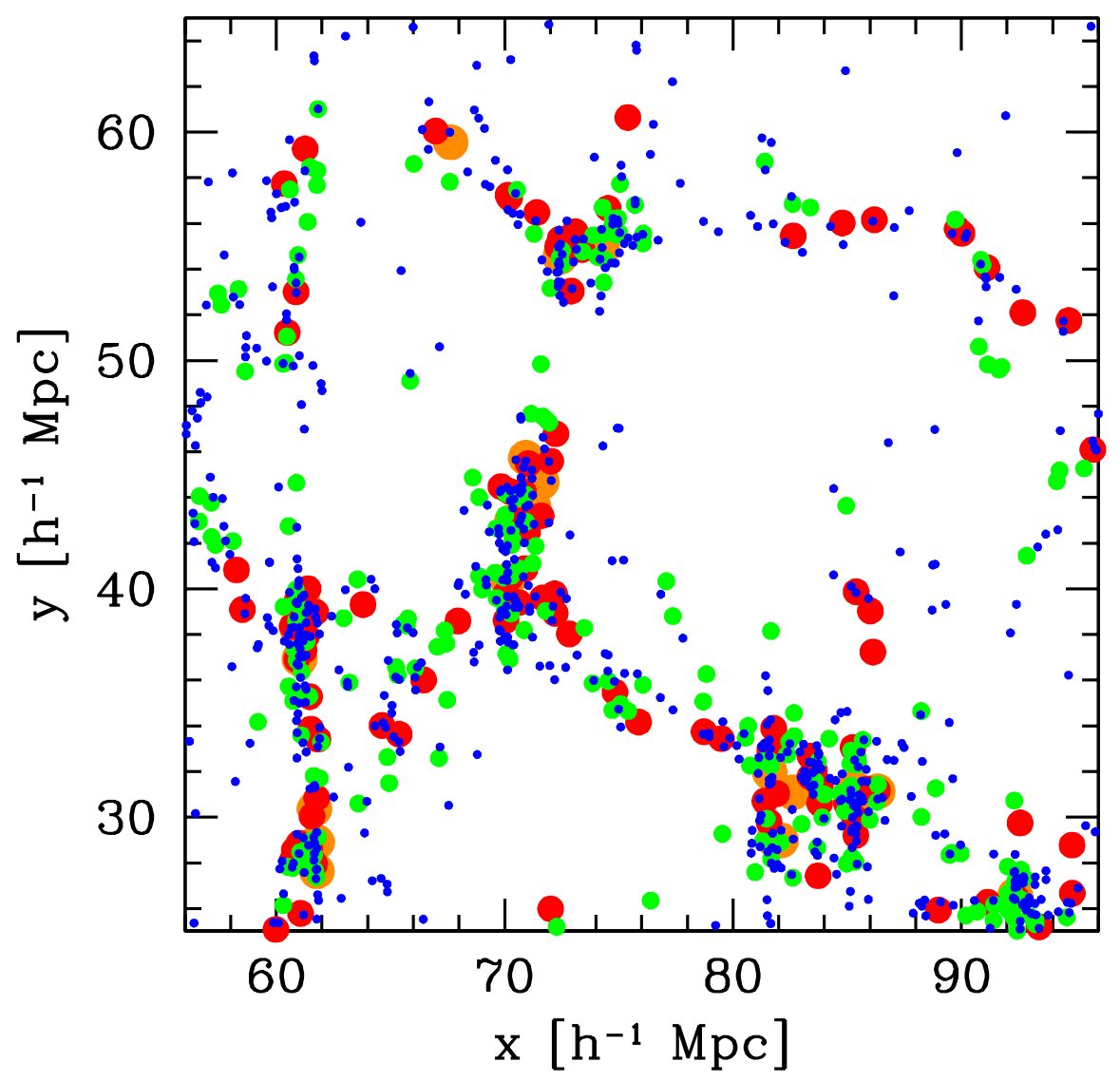

FIG. 1. - Slice through the galaxy population created from the halos within the $96 h^{-1} \mathrm{Mpc}$ simulation. The depth of the slice is $6 h^{-1} \mathrm{Mpc}$. The point size scales with the luminosity of the galaxies. $r$-band magnitudes are as follows: blue $=-14$ and -15 ; green $=-16$ and -17 , red $=-18$ and -19 , orange are all galaxies -20 and brighter.

Figure 2 compares the void luminosity function of our HOD model to the Hoyle et al. (2005) measurements. Our model is in excellent agreement with the data, not only reproducing the overall abundance of void galaxies but also the decrease in the value of $M_{*}$ in the void luminosity function relative to the overall luminosity function.

Interestingly, we find some void galaxies with magnitudes as bright as $M_{r}=-21.5$, just as measured in Hoyle et al. These objects are not scattered into void regions due to redshift space distortions, but are in intrinsically low-density regions as defined by the $M_{r}<-20.5$ galaxies. The minimum mass scale for $M_{r}<-21.5$ galaxies is $\sim 1.8 \times 10^{13} h^{-1} \mathrm{M}_{\odot}$, which are never found in $\delta \lesssim-0.6$ regions in the dark matter distribution. When using the dark matter particles to obtain the local density around each galaxy and recalculate the void luminosity function, there are no void galaxies this bright. Thus, bright void galaxies are due to stochastic biasing of $L_{*}$ galaxies, both from Poisson fluctuations in the number of halos and fluctuations in the number of $L>L_{*}$ galaxies per halo. This creates a few regions in which the dark matter density is above the density threshold but the galaxy density is below it.

\subsection{Nearest Neighbor Statistics}

P01 used nearest neighbor statistics to probe the relative distribution of "ordinary" galaxies $\left(M_{r} \leq-16\right)$ to dwarf galaxies $\left(M_{r}>-16\right)$. If dwarf galaxies preferentially occupy void regions relative to their brighter counterparts, their distribution of nearest neighbors will show a significant tail out to large neighbor distances $R_{\mathrm{nb}}$. To circumvent the problem of different galaxy samples having different mean space densities, for each test object the nearest neighbor in the control sample is found. This distribution is compared to the distribution of $R_{\mathrm{nb}}$ of the control sample to itself. P01 found that the distribution of nearest neighbors for test and control objects are essentially the same, indicating that dwarfs avoid the voids defined by the ordinary objects.

In Figure 3 we present several examples of the cumulative distributions of $R_{\mathrm{nb}}$ taken from L96. Here we have set our control sample to be $M_{r}=[-16,-18)$, and our test sample are galaxies with $M_{r}=[-14,-16)$. The control and test samples are substantially different in terms of their space densities, but are not too dissimilar in the halo masses that they probe. To test any systematics due to these choices we have an additional sample of "bright" objects containing all galaxies $M_{r}<-18$. The largest sample in P01 is roughly 1000 $\left(h^{-1} \mathrm{Mpc}\right)^{3}$, so we have broken our mock galaxy distribution into $9^{3}$ equal-volume cubes of $1225\left(h^{-1} \mathrm{Mpc}\right)^{3}$ to test for cosmic variance in this statistic. The bottom right panel shows the results from the full simulation. We define $R_{t c}$ as the distance to the nearest control galaxy for each test galaxy, while $R_{b c}$ is the distance to the nearest control galaxy for each bright galaxy, and $R_{c c}$ is the distance for each control galaxy to the 


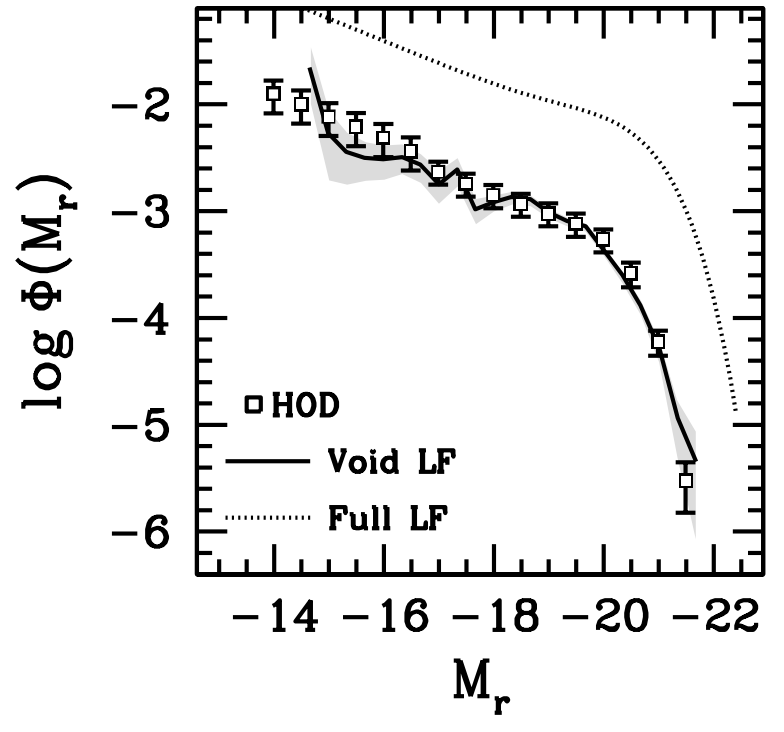

FIG. 2.- Comparison between the luminosity function of void galaxies measured by Hoyle et al. (2005) and predicted by our HOD model. The HOD measurement, shown with the open squares, is a weighted average of results from the $96 h^{-1} \mathrm{Mpc}$ and $192 h^{-1} \mathrm{Mpc}$ simulations (noting that all points at $M_{r}>-17$ are from the $96 h^{-1} \mathrm{Mpc}$ simulation). Errors are estimated by jackknife sampling of each simulation into octants. The solid line is the Hoyle et al. data, with the shaded region representing their error bars. For comparison, the full luminosity function of all galaxies from Blanton et al. (2005) is shown with the dotted curve.

nearest galaxy within the control sample.

The shaded region in each panel of Figure 3 approximates the results from the largest dwarf sample from P01 (his Figure 4 , top set of curves). The upper limit of the shaded region is set by $f\left(<R_{t c}\right)$ while the lower edge is $f\left(<R_{c c}\right)$. P01 determined the ratio of mean $R_{\mathrm{nb}}$ values to be $\left\langle R_{t c}\right\rangle /\left\langle R_{c c}\right\rangle=1.1$. This is nearly identical to the overall value of 1.06 obtained from the L96 simulation. The scatter in this statistic is quite large, with a variance in mean distance ratios over the $9^{3}$ subsamples of 0.23. In many examples in Figure 3, $f\left(<R_{c c}\right)$ extends to distances significantly beyond $f\left(<R_{t c}\right)$. Comparing the $R_{t c}$ distributions to the nearest neighbor statistics for the bright objects to the test sample, $R_{b c}$, yields similar results. The mean distance ratio is 1.19 with a scatter of 0.55 , owing to the lower number density of brighter objects. While the agreement between model and data are encouraging, it is likely fortuitous given the large cosmic scatter in the P01 measurements. However, we conclude that the prediction of the model supports the picture that dwarf galaxies and brighter galaxies have similar spatial distributions in low-density regions. The slight difference in nearest neighbor distributions between dwarf and regular galaxies is also in agreement with the observational results of Lee et al. (2000).

\subsection{Void Probabilities as a Function of Luminosity}

The void probability function (VPF, denoted $P_{0}(r)$ ) is defined as the probability that a randomly placed sphere of radius $r$ contains no galaxies. In Tinker et al. (2007a) we measured the VPF for galaxies as faint as $M_{r}=-19$ from Data Release Four of the Sloan Digital Sky Survey Adelman-McCarthy et al. 2006). Fainter samples were not used because cosmic variance errors become large and galaxy clustering measurements are not available at $M_{r}>-18$. In Figure 4 we present new measurements of the VPF from DR6. The measurements are in magnitude bins, 1 magnitude wide (referenced by their lower limit). We follow the procedures outlined in detail in Tinker et al. (2007a) for both measurements and for comparing the HOD predictions to the data. We use the HOD mocks to estimate error bars on the data, also discussed in Tinker et al. (2007a). The increased sky coverage of DR6 attenuates (but does not eliminate) the cosmic variance considerations for fainter samples. Though we are not able to probe void statistics for dwarf galaxies (in our definition), we are able to make VPF measurements down to $M_{r}=-17$, a two magnitude improvement on our previous results. We use these data to test the robustness of our HOD model and to extend the conclusions of Tinker et al. (2007a) discussed in $\S 1$ to lower luminosities.

Rather than presenting VPFs for each luminosity bin, we consolidate the results by presenting void probabilities at a fixed distance scale as a function of luminosity. The open circles in Figure 4 show $P_{0}(r)$ at the mean intergalactic separation for each magnitude bin, $r=l_{g} \equiv \bar{n}_{g}^{-1 / 3}$. Squares and triangles represent $P_{0}(r)$ at $r=1.3 l_{g}$ and $r=1.6 l_{g}$, respectively. At luminosities below $L_{*}$, the void probability at any multiple of $l_{g}$ is essentially independent of luminosity. Brighter galaxies have a lower probability of finding a void, thus, in the scaled distance $r / l_{g}$, the brightest galaxies have the smallest voids. This has also been seen in the $2 \mathrm{dFGRS}$ data (von Benda-Beckmann \& Müller 2008). This is partially a result of the fact that brighter galaxies have fewer satellite galaxies (a higher satellite fraction results in an increase in $M_{\min }$ in order to match the number density of the galaxy sample), but it is mainly due to the fact that $l_{g}$ for samples on the exponential tail of the of the luminosity function increases much more rapidly than the bias of those galaxies. For $M_{r}<-22$ galaxies, $l_{g}=33 h^{-1} \mathrm{Mpc}$.

The HOD model predictions are shown in Figure 4 for all three simulations. The difference in the amplitude of $P_{0}(r)$ between L96 and the larger simulations is consistent with cosmic variance due to the small volume. Although the model is not calibrated on clustering measurements as done in Tinker et al. (2007a), it matches the observed void probabilities as a function of luminosity and scale. The constraints on $M_{\min }$ are driven primarily by $\bar{n}_{g}$ rather than two-point clustering, therefore $M_{\min }$ and the resulting VPF are similar between Tinker et al. (2007a) and the model presented here.

Although we cannot probe the VPF for galaxies as faint as $M_{r}=-14$ the success of our model in matching $P_{0}(r)$ down to $M_{r}=-17$, the void luminosity function down to $M_{r}=-14.5$, and nearest neighbor statistics down to $M_{r}=-14$ strongly suggest that our extrapolation of the HOD below halo masses of $10^{11} h^{-1} \mathrm{M}_{\odot}$ is robust, and the resulting galaxy distribution represents a complete picture of the structure within underdense regions.

\subsection{The galaxy structure within voids}

In the VPF results of Figure 4, $P_{0}\left(1.6 l_{g}\right) \sim$ constant for $-17 \geq M_{r} \geq-20$ galaxies in both the model and the data. In this magnitude range, voids are self-similar. At fainter magnitudes, the void probability monotonically increases with decreasing brightness. This implies that voids are not selfsimilar for dwarf galaxies, and that the structure of the comic web itself plays a role in the distribution of dwarfs in voids 


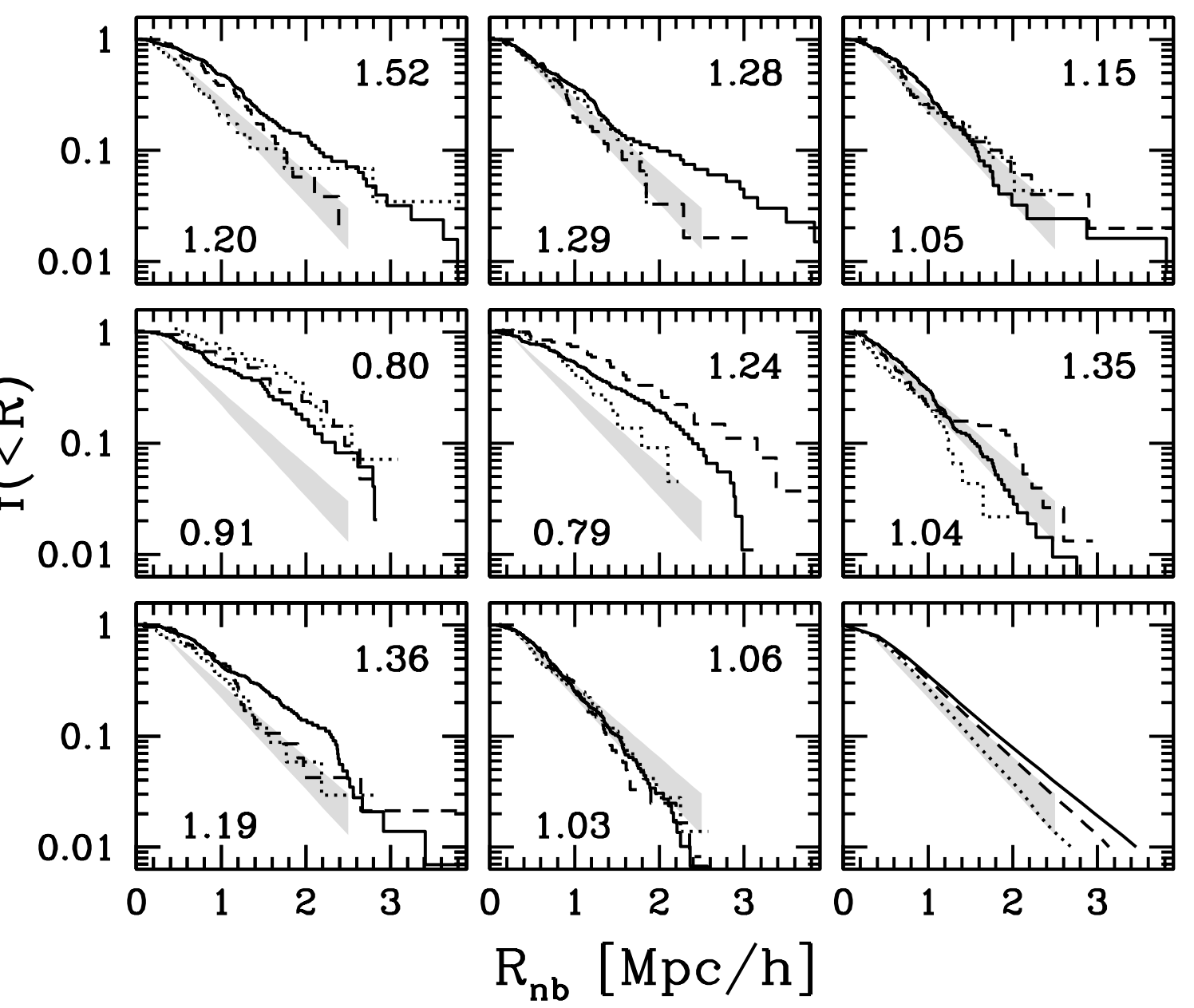

FIG. 3.- Nearest neighbor statistics for "test" galaxies, $M_{r}=[-14,-16)$ (solid histograms), "control" galaxies, $M_{r}=[-16,-18)$, (dashed histograms), and "bright" galaxies, $M_{r}<-18$, (dotted histograms). For each galaxy in a sample, $R_{\mathrm{nb}}$ is the distance to the nearest control galaxy in redshift space. The first eight panels represent random subvolumes of the $96 h^{-1} \mathrm{Mpc}$ simulation that match the volume of the sample in P01. In each panel, the number in the bottom left is the ratio of mean $R_{\mathrm{nb}}$ values, $\left\langle R_{t c}\right\rangle /\left\langle R_{c c}\right\rangle$. The number in the top right is $\left\langle R_{t c}\right\rangle /\left\langle R_{b c}\right\rangle$. The bottom right panel are the overall statistics for the entire box. The shaded region represents the observational results from P01.

(as seen in Figure 1).

The results of Figure 1 and the trend in $P_{0}(r)$ for $M_{r} \geq-17$ galaxies can be interpreted through the change in the halo mass function within the void itself. Figure 5 plots the maximum halo mass as a function of distance from the center of the three deepest voids in L96 (all with radii $\sim 10 \mathrm{~h}^{-1} \mathrm{Mpc}$, and central densities $\delta<-0.9$ ). At $10 h^{-1} \mathrm{Mpc}$ from the center of the void, halos between $10^{12}-10^{13} h^{-1} \mathrm{M}_{\odot}$ can be found. These halos house the $L_{*}$ galaxies that 'define' the edge of the void. But between $10 h^{-1} \mathrm{Mpc}$ and $5 h^{-1} \mathrm{Mpc}$ from the void center, the maximum halo mass drops nearly three orders of magnitude. At the very centers of the voids, only $M \sim 10^{9}$ $h^{-1} \mathrm{M}_{\odot}$ halos are present. The points in Figure 5] show $M_{\min }$ as a function of luminosity from $M_{r}<-21$ to $M_{r}<-10$ samples $^{2}$. The points are placed along the curve to demonstrate how large the void would be in each luminosity. The steep drop in the maximum mass at $R \sim 6 h^{-1} \mathrm{Mpc}$ causes the voids

${ }^{2}$ We caution that the Blanton et al. (2005) luminosity function is calibrated only down to -12 , so results at lower luminosities are an extrapolation. to have sharp boundaries in the galaxy distribution over a wide range of galaxy luminosity. Figure 5 implies that between 5 and $6 h^{-1} \mathrm{Mpc}$ from the void center, the brightest galaxy luminosity will plummet by nearly 5 magnitudes. The galaxies in the inner half of the void (if there are any) would be well below the magnitude limit of the ORS data with which P01 calculate their statistics. Peebles (2007) finds that the abrupt transition into the local void, inside of which no galaxies are observed, occurs at $R \sim 5-7 h^{-1} \mathrm{Mpc}$ from the void center. He further demonstrates that this boundary is nearly identical for galaxies divided into faint and bright samples (with a threshold of $M_{B}=-18$ ). The scatter between halo mass and galaxy luminosity smooths out the slight change in void radius with $M_{r}$ seen in Figure 5. Thus for a luminosity threshold cut, the edge of the local void should be nearly the same for the two samples.

The dashed curve in Figure 5 indicates the critical mass threshold below which Hoeft et al. (2006) find that photoionization significantly reduces the ability of void halos to form 


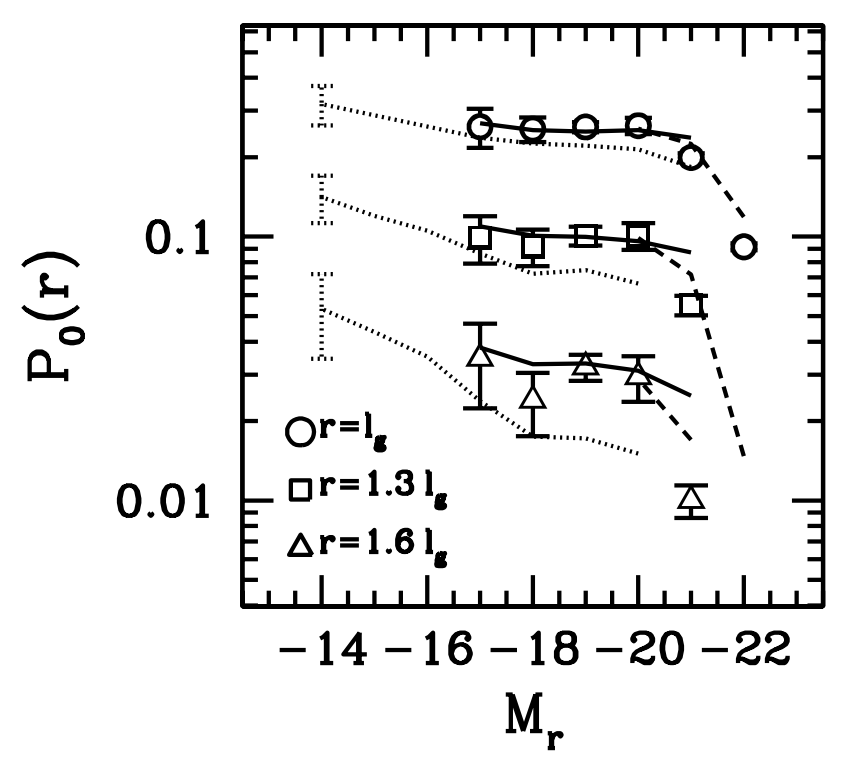

FIG. 4. - The void probability as a function of luminosity at three different separations relative to the mean intergalactic separation, $l_{g}=\bar{n}_{g}^{-1 / 3}$. Points with errors are results from SDSS DR6. Curves represent results from the HOD model from the simulations listed in Table 2: L96 (dotted curves), L192 (solid curves), L384 (dashed curves). Data points for the $M_{r}=-22$ sample are not shown for $r \geq 1.3 l_{g}$ because the VPF is not measured at those distances.

stars. No halos at $R \leq 5 h^{-1} \mathrm{Mpc}$ are above this line, suggesting that the inner half of deep voids will contain no galaxies at all, becoming truly dark. Gottlöber et al. (2003) calculated the mean void mass function for all halos within $80 \%$ of the void radius, finding good agreement with the analytic predictions of Sheth \& Tormen (2002). However, they also find that the shape of the void mass function depends on position within the void itself. The mean void mass function in the L96 simulation (within $0.8 R_{\text {void }}$ ) is in good agreement with their results.

It is important to consider scatter between mass and luminosity for central galaxies. The points in Figure 5 represent $M_{\text {min }}$, the mass at which the halo has only a $50 \%$ probability of containing a galaxy of that luminosity or brighter. The error bars on each point indicate the mass at which that probability is $10 \%$ and if we double the scatter between mass and luminosity to 0.3 . Random fluctuations, or larger physical scatter between mass and luminosity, may place a galaxy slightly further toward the center of the void (or further away), but not enough to smooth out the sharp transition between filament and void seen in the simulations.

\section{SUMMARY AND DISCUSSION}

The void phenomenon consists of two observational facts: that voids contain few, if any, low-luminosity galaxies, and that the few void objects tend to have similar properties to the overall galaxy population. The controversial aspect is whether these facts are at odds with the current cosmology. Although the depth of voids and homogeneity of void objects are striking features of the cosmic web, they are readily explainable within the context of $\Lambda \mathrm{CDM}$, combined with a straightforward model to connect galaxies and dark matter at all luminosities and mass scales.

The simple proposition within our implementation of the

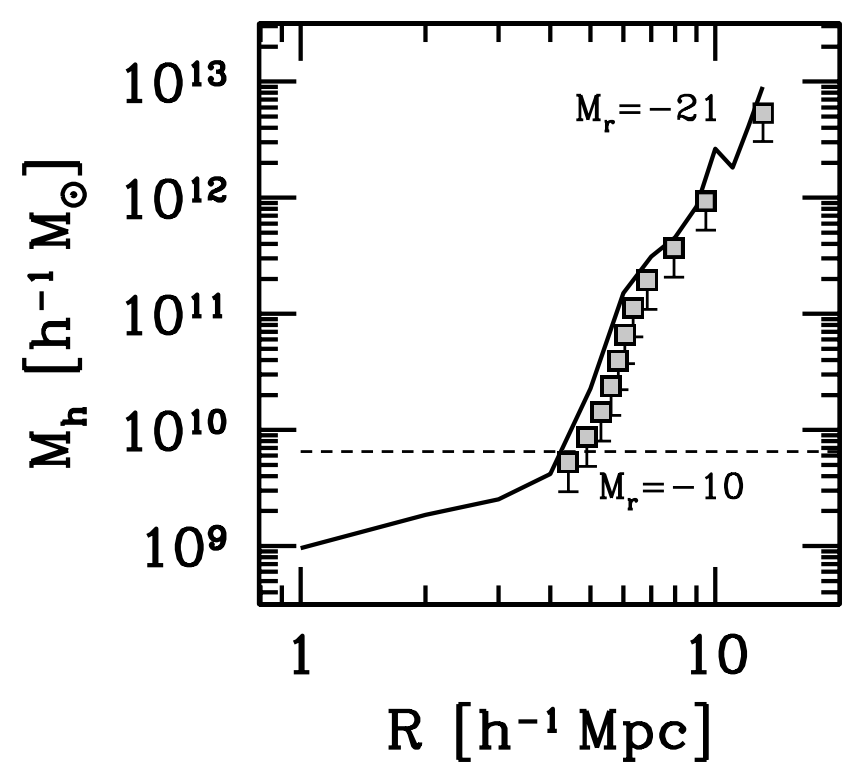

FIG. 5.- The maximum halo mass within voids as a function of distance from the void center. The solid curve represents mean of the largest three voids in the $96 h^{-1} \mathrm{Mpc}$ simulation. The points represent $M_{\min }$ as a function of magnitude, placed along the curve to demonstrate how large the void would be at each luminosity. The dashed curve is the critical mass from Hoeft et al. (2006), below which star formation in void halos is significantly attenuated due to delayed formation histories of void halos.

halo occupation distribution is that galaxy properties are determined solely by the mass of the halo in which the galaxy resides, independent of the halo's larger scale environment ${ }^{3}$ Although this model must break down at some high level of precision or with the details of certain galaxy properties, connecting luminosity to halo mass is a robust method that has passed all tests thus far (Abbas \& Sheth 2006; Skibba et al. 2006; Tinker et al. 2007a, as well as the tests presented in this paper). The mass-only approach to galaxy bias readily explains both aspects of the void phenomenon. First, void galaxies are a fair representation of the field population simply by construction. A galaxy in a $10^{10} h^{-1} \mathrm{M}_{\odot}$ halo does not know if the halo sits in a void or a filament. Recent numerical results have demonstrated that the halo itself does retain information about its environment (the 'assembly bias'; see, e.g., Gao et al. 2005; Wechsler et al. 2006; Gao \& White 2007). However, this effect does not propagate into the void galaxies through either luminosity or color (Tinker et al. 2007a). Patiri et al. (2006b) find that void galaxy properties, even star formation rate or morphology, follow the same distribution as the field. This is echoed in the semi-analytic results of Croton \& Farrar (2008). Therefore, although halos 'remember' their environment, the physics of galaxy formation introduces an intrinsic scatter between halo formation and galaxy properties that washes this signal out.

Second, the lack of dwarf galaxies in voids is a consequence of mapping galaxy luminosity to halo mass in such a way as to preserve the observed abundance of galaxies at each luminosity. The halo mass function scales as $M^{-2}$, while the luminos-

${ }^{3}$ We note that it is possible to create an HOD with depends on environment. The model would then specify $P(N \mid M, \delta)$ rather than $P(N \mid M)$, as done in Tinker et al. (2006, 2007a) and Wechsler et al. (2006). 
ity function scales as roughly $L^{-1}$. This difference in logarithmic slopes implies that the mass to light ratio $M / L$ of galaxies increases as galaxies become fainter. Below $L_{*}$, objects with substantially different luminosities will occupy halos of roughly comparable mass and will trace out the same largescale structure. In order to explain the void phenomenon, we require the physical mechanism that causes the increase in $M / L$ for faint objects (whatever it may be) to apply to all low-mass halos, regardless of environment.

Connecting the luminosity function to the halo mass function naturally predicts that the Tully-Fisher relation becomes flatter at low luminosities. Geha et al. (2006) confirmed this prediction by measuring the rotation velocities of galaxies as faint as $M_{r} \approx-13$. Our model places these galaxies in halos with maximum circular velocities of $\sim 45 \mathrm{~km} \mathrm{~s}^{-1}$, in good agreement with the observed rotation speeds of $35-60 \mathrm{~km} \mathrm{~s}^{-1}$.

As stated above, parameterizing the HOD as a function only of halo mass is only an approximation of the underlying physics (although a surprisingly good approximation). In the context of the void phenomenon, it should be noted that any influence of environment is mostly likely to reduce galaxy formation efficiency in underdense regions. This is the effect of the photoionization arguments in Hoeft et al. (2006), and is also seen in the semi-analytic model of Croton et al.
(2007). A reduction in formation efficiency in voids makes $M_{\min }$ higher at fixed luminosity. There is no need to invoke exotic new physics, such as modified gravity models, to lower the formation efficiency of halos in underdense regions to match the data. Several studies have demonstrated that a long-range scalar potential acting on the dark matter can accelerate the evacuation of matter from voids during the growth of structure (Farrar \& Peebles 2004; Gubser \& Peebles 2004; Nusser et al. 2005). Although comparison of these models to observational data is difficult analytically, numerical simulations of these alternative gravity models, like those by Nusser et al. (2005), can be used in the same manner as the simulations here. The excellent agreement between our HOD model and the observations may place upper limits on such models, since models that produce larger voids at any luminosity would come into conflict with measured void statistics.

We would like to thank Mike Warren for use of his simulations. We also thank Jim Peebles for comments on the manuscript. J.T. was supported by the Chandra award GO56120B and National Science Foundation (NSF) under grant AST-0239759.

\section{REFERENCES}

Abbas, U. \& Sheth, R. K. 2006, MNRAS, accepted, (astro-ph/0601407)

Adelman-McCarthy, J. K. et al. 2006, ApJS, 162, 38

—. 2007, ApJS, submitted (ArXiv/070.3413)

Benson, A. J., Hoyle, F., Torres, F., \& Vogeley, M. S. 2003, MNRAS, 340, 160

Berlind, A. A. \& Weinberg, D. H. 2002, ApJ, 575, 587

Blanton, M. R., Hogg, D. W., Bahcall, N. A., Brinkmann, J., Britton, M. Connolly, A. J., Csabai, I., Fukugita, M., Loveday, J., Meiksin, A., Munn, J. A., Nichol, R. C., Okamura, S., Quinn, T., Schneider, D. P., Shimasaku, K., Strauss, M. A., Tegmark, M., Vogeley, M. S., \& Weinberg, D. H. 2003, ApJ, 592, 819

Blanton, M. R., Lupton, R. H., Schlegel, D. J., Strauss, M. A., Brinkmann, J., Fukugita, M., \& Loveday, J. 2005, ApJ, 631, 208

Bullock, J. S., Kolatt, T. S., Sigad, Y., Somerville, R. S., Kravtsov, A. V., Klypin, A. A., Primack, J. R., \& Dekel, A. 2001, MNRAS, 321, 559

Chen, J. 2007, å, submittied (ArXiv:0712.0003)

Conroy, C., Coil, A. L., White, M., Newman, J. A., Yan, R., Cooper, M. C., Gerke, B. F., Davis, M., \& Koo, D. C. 2005, ApJ, 635, 990

Conroy, C., Wechsler, R. H., \& Kravtsov, A. V. 2006, ApJ, 647, 201

Cooray, A. \& Sheth, R. 2002, Phys. Rep., 372, 1

Croton, D. J., Colless, M., Gaztañaga, E., Baugh, C. M., Norberg, P., Baldry, I. K., Bland-Hawthorn, J., Bridges, T., Cannon, R., Cole, S., Collins, C. Couch, W., Dalton, G., de Propris, R., Driver, S. P., Efstathiou, G., Ellis, R. S., Frenk, C. S., Glazebrook, K., Jackson, C., Lahav, O., Lewis, I., Lumsden, S., Maddox, S., Madgwick, D., Peacock, J. A., Peterson, B. A. Sutherland, W., \& Taylor, K. 2004, MNRAS, 352, 828

Croton, D. J. \& Farrar, G. R. 2008, ArXiv:0801.2771

Croton, D. J., Gao, L., \& White, S. D. M. 2007, MNRAS, 374, 1303

De Lucia, G., Kauffmann, G., Springel, V., White, S. D. M., Lanzoni, B. Stoehr, F., Tormen, G., \& Yoshida, N. 2004, MNRAS, 348, 333

Diemand, J., Kuhlen, M., \& Madau, P. 2007, ApJ, 657, 262

Farrar, G. R. \& Peebles, P. J. E. 2004, ApJ, 604, 1

Furlanetto, S. R. \& Piran, T. 2006, MNRAS, 366, 467

Gao, L., Springel, V., \& White, S. D. M. 2005, MNRAS, 363, L66

Gao, L. \& White, S. D. M. 2007, MNRAS, 377, L5

Gao, L., White, S. D. M., Jenkins, A., Stoehr, F., \& Springel, V. 2004, MNRAS, 355, 819

Geha, M., Blanton, M. R., Masjedi, M., \& West, A. A. 2006, ApJ, 653, 240

Gottlöber, S., Łokas, E. L., Klypin, A., \& Hoffman, Y. 2003, MNRAS, 344, 715

Gregory, S. A. \& Thompson, L. A. 1978, ApJ, 222, 784

Gubser, S. S. \& Peebles, P. J. E. 2004, Phys. Rev. D, 70, 123511

Hoeft, M., Yepes, G., Gottlöber, S., \& Springel, V. 2006, MNRAS, 371, 401

Hoyle, F., Rojas, R. R., Vogeley, M. S., \& Brinkmann, J. 2005, ApJ, 620, 618

Hoyle, F. \& Vogeley, M. S. 2004, ApJ, 607, 751

Kirshner, R. P., Oemler, Jr., A., Schechter, P. L., \& Shectman, S. A. 1981, ApJ, 248, L57

Kravtsov, A. V., Berlind, A. A., Wechsler, R. H., Klypin, A. A., Gottlöber, S., Allgood, B., \& Primack, J. R. 2004, ApJ, 609, 35

Lee, J. C., Salzer, J. J., Law, D. A., \& Rosenberg, J. L. 2000, ApJ, 536, 606

Lin, Y.-T., Mohr, J. J., \& Stanford, S. A. 2004, ApJ, 610, 745

Mathis, H. \& White, S. D. M. 2002, MNRAS, 337, 1193
Navarro, J. F., Frenk, C. S., \& White, S. D. M. 1997, ApJ, 490, 493

Nusser, A., Gubser, S. S., \& Peebles, P. J. 2005, Phys. Rev. D, 71, 083505

Padmanabhan, N., White, M., Norberg, P., \& Porciani, C. 2008, ArXiv eprints, 802

Patiri, S. G., Betancort-Rijo, J. E., Prada, F., Klypin, A., \& Gottlöber, S. 2006a, MNRAS, 369, 335

Patiri, S. G., Prada, F., Holtzman, J., Klypin, A., \& Betancort-Rijo, J. 2006b, MNRAS, 372, 1710

Peebles, P. J. E. 2001, ApJ, 557, 495

-. 2007, ArXiv:0712.2757

Santiago, B. X., Strauss, M. A., Lahav, O., Davis, M., Dressler, A., \& Huchra J. P. 1995, ApJ, 446, 457

Scoccimarro, R., Sheth, R. K., Hui, L., \& Jain, B. 2001, ApJ, 546, 20

Seljak, U. 2000, MNRAS, 318, 203

Sheth, R. K. \& Tormen, G. 2002, MNRAS, 329, 61

Sheth, R. K. \& van de Weygaert, R. 2004, MNRAS, 350, 517

Skibba, R., Sheth, R. K., Connolly, A. J., \& Scranton, R. 2006, MNRAS, 369 68

Springel, V., White, S. D. M., Jenkins, A., Frenk, C. S., Yoshida, N., Gao, L., Navarro, J., Thacker, R., Croton, D., Helly, J., Peacock, J. A., Cole, S., Thomas, P., Couchman, H., Evrard, A., Colberg, J., \& Pearce, F. 2005, Nature, 435, 629

Tinker, J. L. Conroy, C., Norberg, P., Patiri, S., Weinberg, D. H., \& Warren, M. S. 2007a, ApJ, submitted, (arxiv:0707.3445)

Tinker, J. L., Kravtsov, A. V., Klypin, A., Abazajian, K., Warren, M. S., Yepes, G., Gottlober, S., \& Holz, D. E. 2008, ApJ, submitted (ArXiv:0803.2706)

Tinker, J. L., Norberg, P., Weinberg, D. H., \& Warren, M. S. 2007b, ApJ, 659, 877

Tinker, J. L., Weinberg, D. H., \& Warren, M. S. 2006, ApJ, 647, 737

van den Bosch, F. C., Yang, X., Mo, H. J., Weinmann, S. M., Macciò, A. V.,

More, S., Cacciato, M., Skibba, R., \& Kang, X. 2007, MNRAS, 376, 841

Vogeley, M. S., Geller, M. J., Park, C., \& Huchra, J. P. 1994, AJ, 108, 745

von Benda-Beckmann, A. M. \& Müller, V. 2008, MNRAS, 65

Warren, M. S., Abazajian, K., Holz, D. E., \& Teodoro, L. 2006, ApJ, 646, 881

Warren, M. S. \& Salmon, J. K. 1993, in Supercomputing '93, IEEE Comp. Soc.

Wechsler, R. H., Zentner, A. R., Bullock, J. S., Kravtsov, A. V., \& Allgood, B. 2006, ApJ, 652, 71

Weinberg, D. H., Colombi, S., Davé, R., \& Katz, N. 2006, ApJ, submitted (astro-ph/0604393)

White, M., Zheng, Z., Brown, M. J. I., Dey, A., \& Jannuzi, B. T. 2007, ApJ, 655, L69

Zehavi, I., Weinberg, D. H., Zheng, Z., Berlind, A. A., Frieman, J. A., Scoccimarro, R., Sheth, R. K., Blanton, M. R., Tegmark, M., Mo, H. J., et al. 2004, ApJ, 608, 16 
Zehavi, I., Zheng, Z., Weinberg, D. H., Frieman, J. A., Berlind, A. A., Blanton, M. R., Scoccimarro, R., Sheth, R. K., Strauss, M. A., Kayo, I., Suto, Y., Fukugita, M., Nakamura, O., Bahcall, N. A., Brinkmann, J., Gunn, J. E., Hennessy, G. S., Ivezić, Ž., Knapp, G. R., Loveday, J., Meiksin, A., Schlegel, D. J., Schneider, D. P., Szapudi, I., Tegmark, M., Vogeley, M. S., \& York, D. G. 2005, ApJ, 630, 1

Zentner, A. R., Berlind, A., Bullock, J. S., Kravtsov, A., \& Wechsler, R. H. 2005, ApJ, 624, 505
Zheng, Z., Berlind, A. A., Weinberg, D. H., Benson, A. J., Baugh, C. M., Cole, S., Davé, R., Frenk, C. S., Katz, N., \& Lacey, C. G. 2005, ApJ, 633, 791

Zheng, Z., Coil, A. L., \& Zehavi, I. 2007, ApJ, submitted, (astro-ph/0703457) 